\title{
OSIRIS-REx, Returning the Asteroid Sample
}

\author{
Thomas Ajluni \\ ASRC Federal Space \& Defense \\ 7000 Muirkirk Meadows Drive \\ Suite 100, Beltsville, MD 20705 \\ 301.286.1831 \\ thomas.m.ajluni@nasa.gov \\ David Everett \\ NASA Goddard Space Flight \\ Center, 8800 Greenbelt Road \\ Greenbelt, MD 20771 \\ 301.286.1596 \\ david.f.everett@nasa.gov
}

\author{
Timothy Linn \\ Lockheed Martin Space Systems \\ Company, P.O. Box 179 \\ Denver, CO 80201 \\ 303-977-0659 \\ timothy.m.linn@lmco.com \\ Ronald Mink \\ NASA Goddard Space Flight \\ Center, 8800 Greenbelt Road \\ Greenbelt, MD 20771 \\ 301.286.3524 \\ ronald.g.mink@nasa.gov
}

\author{
William Willcockson \\ Lockheed Martin Space Systems \\ Company, P.O. Box 179 \\ Denver, CO 80201 \\ 303-977-5094 \\ william.h.willcockson@Imco.com \\ Joshua Wood \\ Lockheed Martin Space Systems \\ Company, P.O. Box 179 \\ Denver, CO 80201 \\ 303-977-3199 \\ joshua.l.wood@lmco.com
}

\begin{abstract}
This paper addresses the technical aspects of the sample return system for the upcoming Origins, Spectral Interpretation, Resource Identification, and Security-Regolith Explorer (OSIRIS-REx) asteroid sample return mission. The overall mission design and current implementation are presented as an overview to establish a context for the technical description of the reentry and landing segment of the mission.
\end{abstract}

The prime objective of the OSIRIS-REx mission is to sample a primitive, carbonaceous asteroid and to return that sample to Earth in pristine condition for detailed laboratory analysis. Targeting the near-Earth asteroid Bennu, the mission launches in September 2016 with an Earth reentry date of September 24, 2023.

OSIRIS-REx will thoroughly characterize asteroid Bennu providing knowledge of the nature of near-Earth asteroids that is fundamental to understanding planet formation and the origin of life. The return to Earth of pristine samples with known geologic context will enable precise analyses that cannot be duplicated by spacecraft-based instruments, revolutionizing our understanding of the early Solar System. Bennu is both the most accessible carbonaceous asteroid and one of the most potentially Earth-hazardous asteroids known. Study of Bennu addresses multiple NASA objectives to understand the origin of the Solar System and the origin of life and will provide a greater understanding of both the hazards and resources in near-Earth space, serving as a precursor to future human missions to asteroids.

This paper focuses on the technical aspects of the Sample Return Capsule (SRC) design and concept of operations, including trajectory design and reentry retrieval. Highlights of the mission are included below.

The OSIRIS-REx spacecraft provides the essential functions for an asteroid characterization and sample return mission:
- attitude control

- propulsion

- power

- thermal control

- telecommunications

- command and data handling

- $\quad$ structural support to ensure successful rendezvous with Bennu

- characterization of Bennu's properties

- delivery of the sampler to the surface, and return of the spacecraft to the vicinity of the Earth

- $\quad$ sample collection, performed by the Touch-and-Go Sample Acquisition Mechanism (TAGSAM), to acquire a regolith sample from the surface

- $\quad$ Earth re-entry and SRC recovery

Following sample collection, OSIRIS-REx drifts away from Bennu until the Asteroid Departure Maneuver is commanded on March 4, 2021, sending OSIRIS-REx on a ballistic return cruise to Earth. No additional large deterministic maneuvers are required to return the SRC to Earth. During the cruise, tracking and trajectory correction maneuvers (TCMs) are performed as necessary to precisely target the entry corridor. As OSIRIS-REx approaches Earth, the reentry plans are reviewed starting about a year before arrival, and preparations begin. The spacecraft is targeted away from the Earth until 7 days before entry. The final two trajectory correction maneuvers bring the spacecraft on target toward the Utah Test and Training Range (UTTR), with sufficient time for contingency resolution. The SRC releases 4 hours prior to atmospheric entry interface and, using the Stardust capsule heritage design, employs a traditional drogue and main parachute descent system for a soft touchdown. 


\section{TABLE OF CONTENTS}

1. INTRODUCTION .2

2. MISSION DESIGN. 3

3. SAMPLE AND RETURN CAPSULE DESIGN ..........7

4. SAMPLE COLLECTION ......................................9

5. RETURN CRUISE ..............................................12

6. EARTH RETURN AND SRC RECOVERY ..........12

7. SUMMARY ....................................................15

REFERENCES.....................................................15

\section{INTRODUCTION}

\section{The Asteroid}

OSIRIS-REx will thoroughly characterize asteroid Bennu (Figure 1-1). Knowledge of the nature of near-Earth asteroids is fundamental to understanding planet formation and the origin of life. The return to Earth of pristine samples with known geologic context will enable precise analyses that cannot be duplicated by spacecraft-based instruments, revolutionizing our understanding of the early Solar System. Bennu is both the most accessible carbonaceous asteroid and one of the most potentially Earth-hazardous asteroids known. As a B-type carbonaceous asteroid, Bennu represents an important source of volatiles and organic matter to Earth as well as being a direct remnant of the original building blocks of the terrestrial planets. Its properties have been well characterized by ground- and space-based telescopes in the visible, infrared and radar, greatly reducing mission risk and providing strong evidence for the presence of regolith available for sampling. Its relatively Earth-like, low delta-V orbit is conducive for a low energy New Frontiers-level mission.

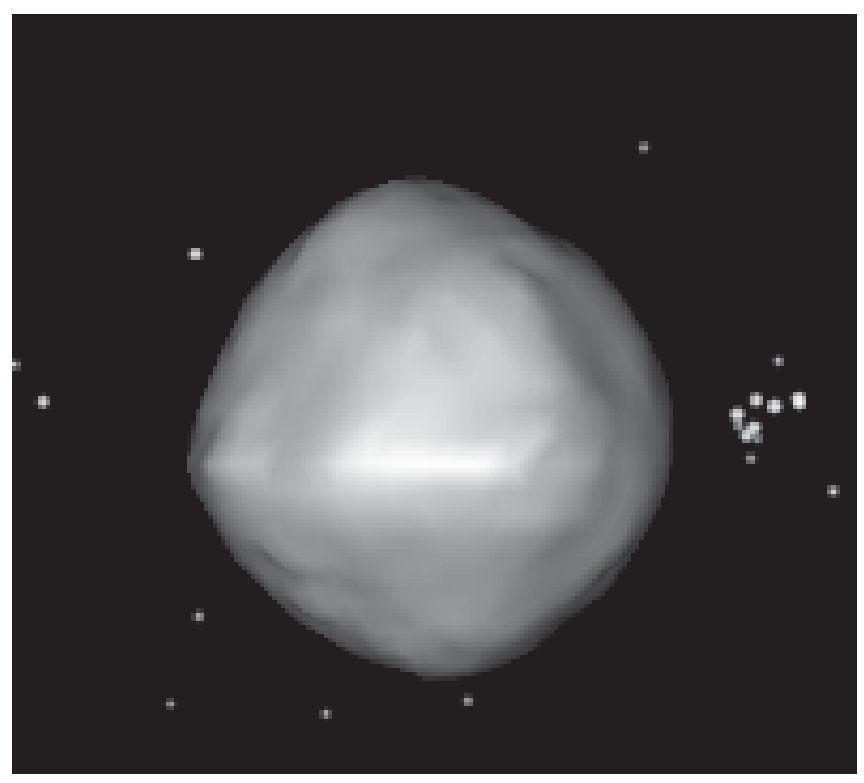

Figure 1-1The Asteroid Bennu
Study of Bennu addresses multiple NASA objectives to understand the origin of the Solar System and the origin of life and will provide a greater understanding of both the hazards and resources in near-Earth space, serving as a precursor to future human missions to asteroids.

\section{Getting There}

OSIRIS-REx launches in September 2016 on an Atlas V 411 from Cape Canaveral Air Station with a backup launch period occurring one year later. Following an Earth flyby and gravity assist in Sept 2017, OSIRIS-REx cruises for 11 months and starts the optical search for Bennu in August 2018, marking the beginning of the Approach phase. Rendezvous occurs in October 2018, followed by a month of slow approach to allow the flight system to search for moons around Bennu and to refine its shape and spin state models. Time at Bennu includes 505 days of operations followed by 426 days of quiescent operations time that serves as contingency before the first Bennu departure opportunity in March of 2021.

\section{The Science}

Beginning with the Preliminary Survey and Orbital A phases in November 2018, OSIRIS-REx lays the foundation for navigating the spacecraft through the remainder of the encounter by estimating the mass of Bennu and transitioning from star-based to surface landmark-based optical navigation for precisely estimating the spacecraft's state relative to the asteroid. OSIRIS-REx then performs comprehensive global mapping of the texture, mineralogy, and chemistry of Bennu in the Detailed Survey phase, resolving geological features, revealing its geologic and dynamic history, and providing context for the returned samples. The Orbital B phase, beginning in February 2019, establishes the "Safe Home" orbit from which all sorties closer to the surface begin. In the Reconnaissance phase, the instruments document the regolith at candidate sampling sites in-situ at scales down to the subcentimeter, providing the high-resolution information needed to confirm the presence of sampleable regolith and to select the primary sample site. Following site selection, a sequence of Touch-and-Go (TAG) rehearsal steps are conducted to separately demonstrate each step in the sample collection sequence prior to sending the flight system to the surface for sampling. In the Sample Collection phase OSIRIS-REx acquires a minimum of $60 \mathrm{~g}$ of bulk regolith and a separate 26 $\mathrm{cm}^{2}$ of fine-grained surface material from Bennu.

\section{Coming Home}

The departure burn from Bennu occurs in March 2021, 931 days after arrival. On September 24, 2023, the Sample Return Capsule (SRC) lands at the Utah Test and Training Range (UTTR). Stardust heritage procedures are followed to transport the SRC to Johnson Space Center (JSC), where the samples are removed and delivered to the OSIRIS-REx curation facility. Analyses of these samples provide unprecedented knowledge about presolar history through the initial stages of planet formation to the origin of life. 


\section{MISSION DESIGN}

\section{Spacecraft}

The OSIRIS-REx spacecraft provides the essential functions for an asteroid characterization and sample return mission: attitude control, propulsion, power, thermal control, telecommunications, command and data handling, and structural support. These subsystems are designed to ensure successful rendezvous with Bennu, characterization of its properties, delivery of the sampler to the surface, and return of the spacecraft to the vicinity of the Earth. Sample collection, performed by the Touch-and-Go Sample Acquisition Mechanism (TAGSAM), acquires a regolith sample from the surface.
Earth re-entry is performed by the Sample Return Capsule (SRC) which delivers the sample to the UTTR. The Flight System block diagram is shown in Figure 2-1. Views of the spacecraft are shown in Figures 2-2, 2-3 and 2-4.

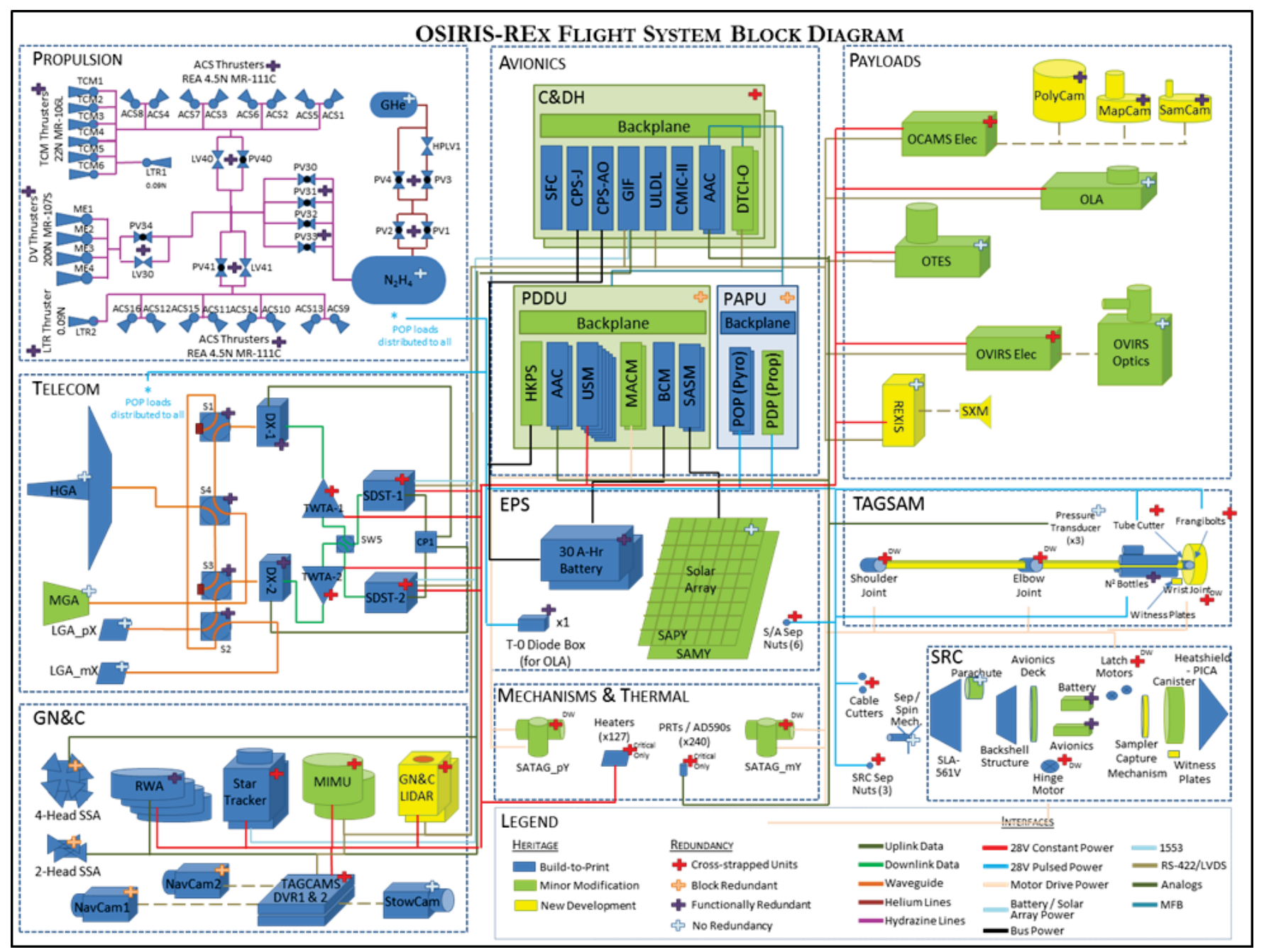

Figure 2-1 Spacecraft Block Diagram 


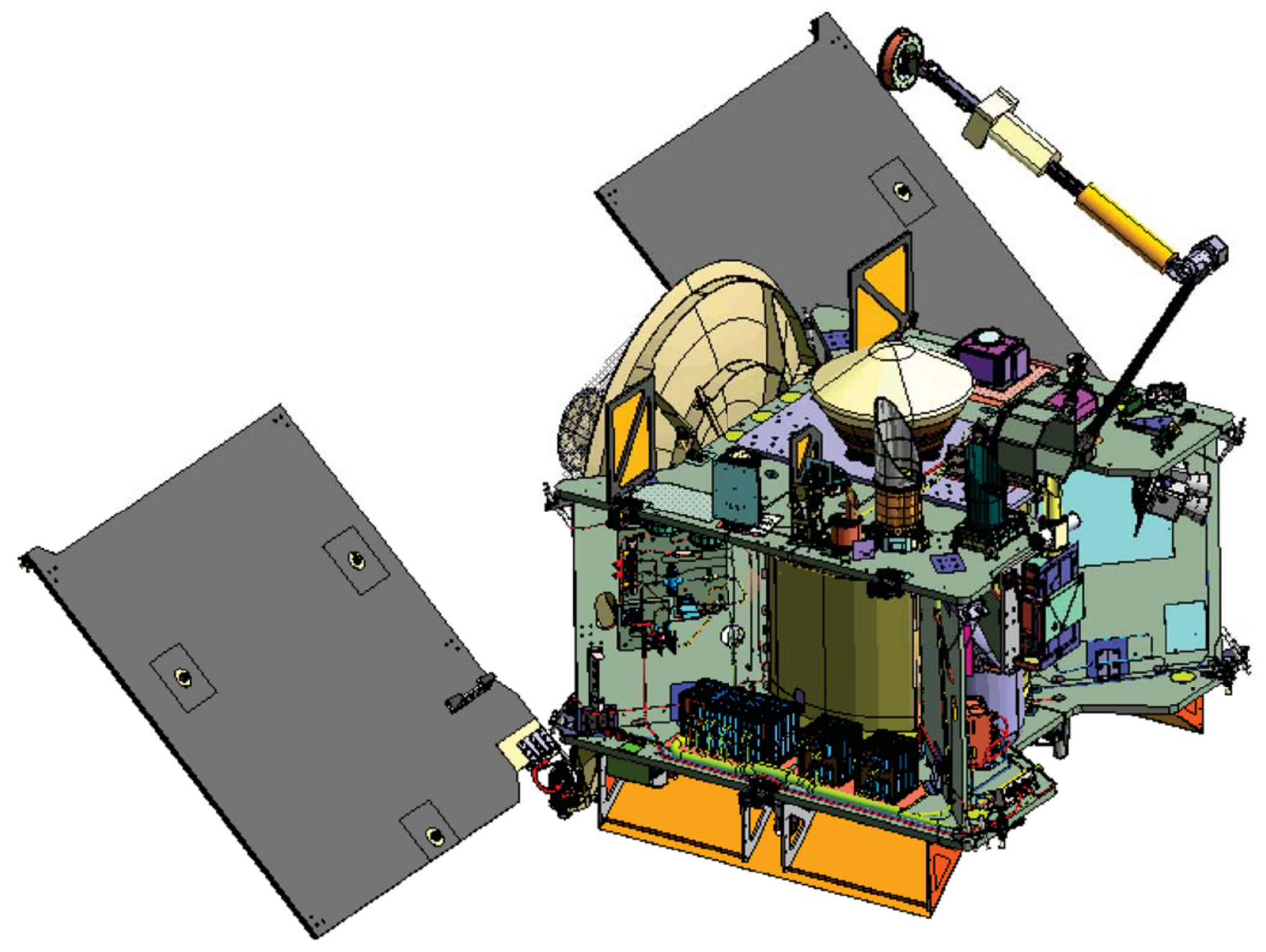

Figure 2-2 Spacecraft Internal Structure Depicting Instruments and Subsytem Components with TAGSAM Arm Deployed 


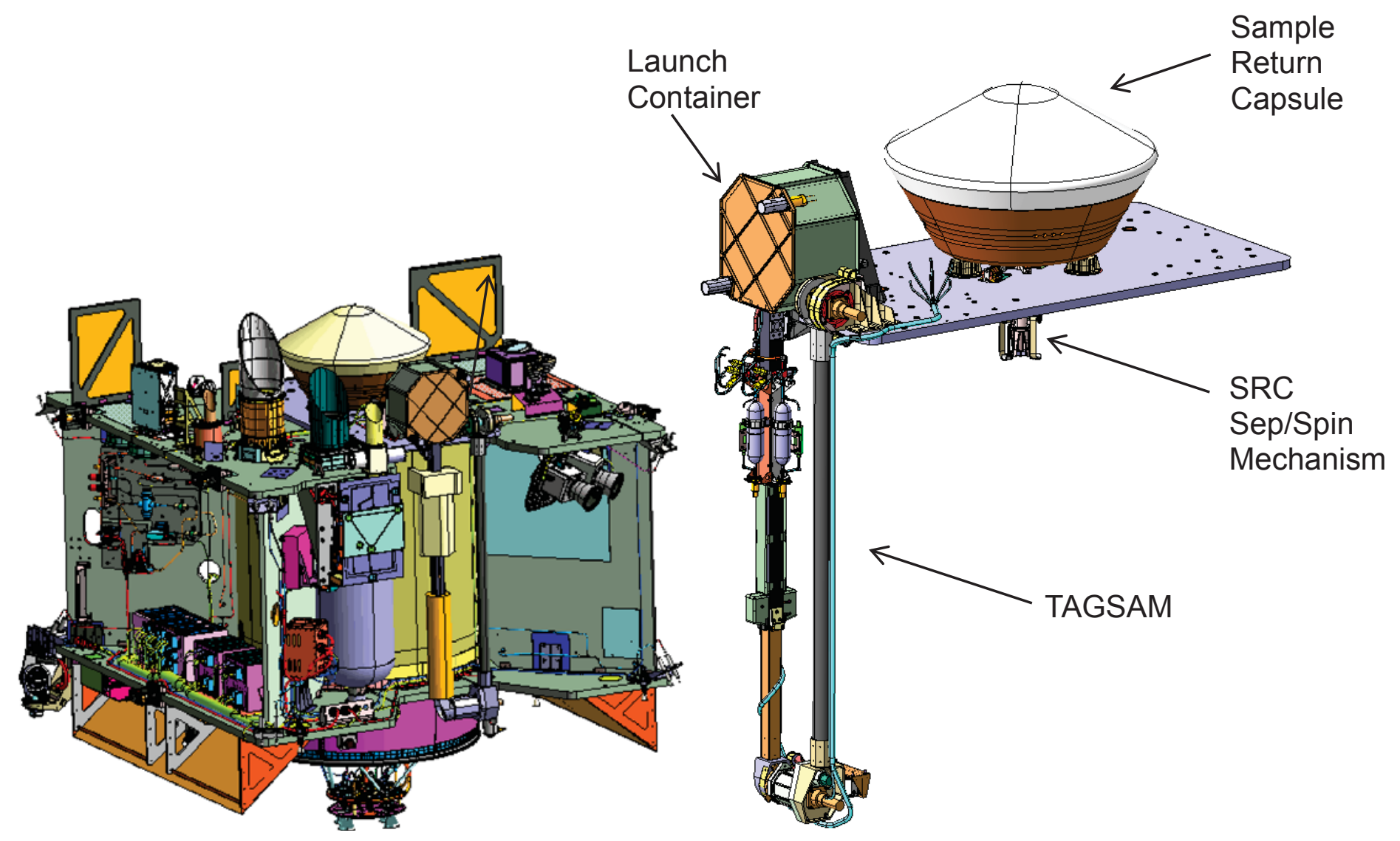

Figure 2-3 Assembled Spacecraft (S/A's Removed)

Figure 2-4 SARA Including SRC and TAGSAM

\section{Science Instruments}

OSIRIS-REx has an extensive array of scientific instruments to both characterize Bennu's physical properties and to provide critical data necessary to support sample acquisition. An array of optical cameras in the visible band (both monochromatic and multispectral) image the asteroid at various ranges, lighting conditions and resolutions as shown in Figure 2-5. A LIDAR system is used to directly measure surface features. Visible, Infrared, and X-ray spectrometers are also used to measure surface constituents and chemical properties. A thermal emission spectrometer is used to measure surface temperatures. The data sets are combined with spacecraft tracking data to determine Bennu's size, mass, orbit and orbit propagation including measurement of the Yarkovsky effect. The primary science objectives are shown below:

Objective 1: Return and analyze a sample of pristine carbonaceous asteroid regolith in an amount sufficient to study the nature, history and distribution of its constituent minerals and organic material.

Objective 2: Map the global properties, chemistry, and mineralogy of a primitive carbonaceous asteroid to characterize its geologic and dynamic history and provide context for the returned samples.
Objective 3: Document the texture, morphology, geochemistry, and spectral properties of the regolith at the sampling site in situ at scales down to the sub-centimeter.

Objective 4: Measure the Yarkovsky effect on a potentially hazardous asteroid and constrain the asteroid properties that contribute to this effect.

Objective 5: Characterize the integrated global properties of a primitive carbonaceous asteroid to allow for direct comparison with ground-based telescopic data of the entire asteroid population.

The Yarkovsky effect is a net force on the asteroid due to the absorption of solar photons followed by the emission of infrared photons after the asteroid has rotated somewhat. The net force will significantly alter the asteroid's orbit over time. This effect is the main source of uncertainty in the prediction of Bennu's orbit into the future. OSIRIS-REx will measure the change in Bennu's orbit and combine that information with the characteristics of the asteroid in order to improve the models of the Yarkovsky effect. This modeling will be applicable to many asteroids. 


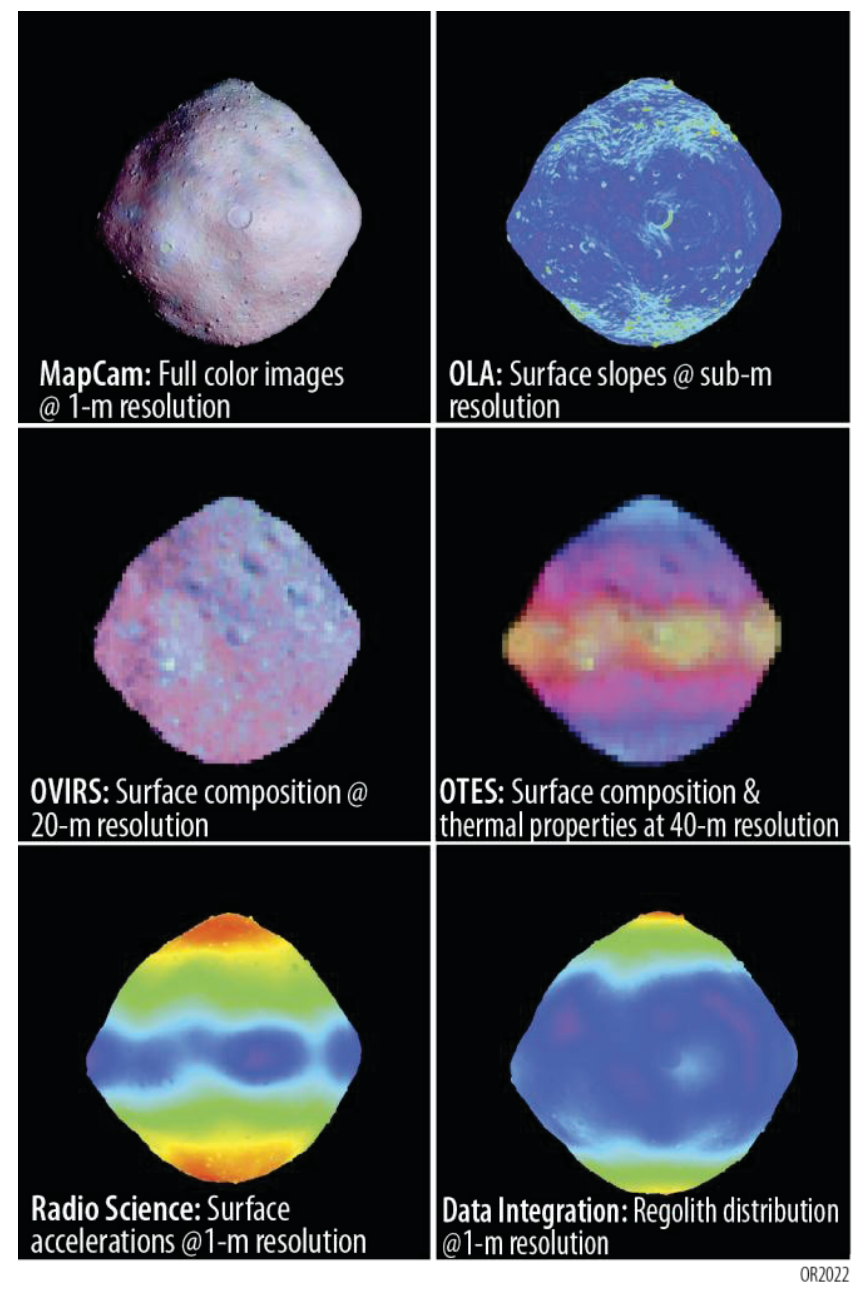

Figure 2-5 Imagery of Bennu is captured in multiple wavelengths and resolutions by a wide array of science instruments 


\section{SAMPLE RETURN CAPSUlE DESIGN}

\section{Overview}

Lockheed Martin (LM) is the prime contractor for the spacecraft, including the SRC (shown in Figure 3-1), and is responsible for overall requirements and design. OSIRISREx is also using NASA expertise at Langley and Ames Research Centers to ensure that the SRC will perform as required for this mission.

Since early in phase B, the SRC team has been developing and refining the aerodynamic models specifically for the OSIRIS-REx trajectory and baseline SRC mass. Modeling includes free molecular, hypersonic, and supersonic analyses and was performed by Langley using the latest Computational Fluid Dynamics (CFD) capability for hypersonic aerodynamics. Langley and LM compared results to earlier work done on Stardust to ensure the models are correct. Langley also has the principal Entry Descent and Landing (EDL) trajectory simulation, incorporating full 6degree-of-freedom (6-DOF) dynamics to calculate aerodynamic forces and SRC motion throughout the atmosphere. This simulation utilizes POST-II architecture that includes capsule and parachute aerodynamics and is based on a similar simulation used for the Stardust reentry mission. The modeling includes the sequencing and triggering logic for events such as the parachute deployment. Initialization for the simulations comes from two sources: the exo-atmospheric trajectory errors are established by the navigation team at KinetX while the capsule spin dynamics are assessed by LM's analysis of the SRC spring separation dynamics.

This simulation incorporates the latest wind data for the UTTR through the use of EarthGRAM-2010's Range Reference Atmosphere capability that is tied to Dugway's meteorological database. Langley has also assessed the dynamics of the returned asteroid regolith sample for its effect on the SRC attitude stability. In conjunction with soil mechanics simulations at LM, the unconstrained soil does not have an adverse effect on the spinning dynamics of the system. LM has performed trajectory verification activity as well as design trajectory development using both a 3-DOF and independent 6-DOF simulation, both also based on POST-II. In parallel with LM's analysis, the two organizations have compared results and investigated discrepancies to ensure the differences are fully understood and not the result of errors in the models. Results from these analyses are used to ensure the SRC remains within the design constraints of the original Stardust qualification and provides a basis for subsequent aerothermal design trajectories which are produced by LM. Also in the flight dynamics realm, Langley is looking at the parachute design and qualification information to ensure that it will meet the OSIRIS-REx requirements, as determined by the modeling.

Full-body hypersonic aeroheating environments are developed by LM with key verification assistance by Ames Research Center. Both organizations use independent CFD analysis and codes (Langley

Aerothermodynamic Upwind Relaxation Algorithm), (LAURA) for LM and Data Parallel Line Relaxation (DPLR) for Ames) which have been exercised in previous reentry programs, most recently in the successful MSL/Curiosity Mars Lander aeroshell design. Where environmental issues have arisen, outside NASA experts have been engaged - most notably in characterizing aft-body radiation heating from the energetic wake flow. Ames is working with the LM team to ensure that the thermal protection of the SRC is sufficient for the mission. Ames has principal analytic responsibility for the high energy Phenolic Impregnated Carbon Ablator (PICA) ablator used in the heatshield while LM is responsible for analysis of the SLA-561V ablator used on the backshell. Both teams start with the aerothermal heating profile results and then model the performance of the heat shield, backshell and other thermal coatings. Ames is also reviewing the PICA development by the vendor to ensure the processes are within the parameters established for Stardust. Arcjet testing of flight thermal protection system witness coupons will be performed at Ames's 60mW Interaction Heating Facility as part of the flight acceptance testing later in 2014.

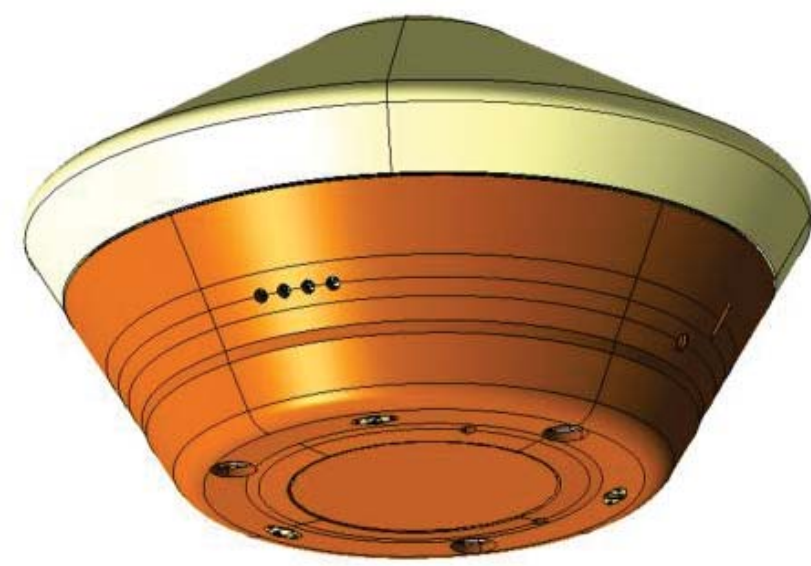

Figure 3-1 Sample Return Capsule (SRC)

\section{SRC Design}

The SRC is quite similar to the Stardust capsule design. Chief differences are in the sample capture and storage. The capsule overall mechanical design, in particular all aspects relating to reentry, are identical to Stardust. Obviously the reuse of a heritage design reduces cost, schedule, and risk for the mission and allows the technical team to focus on the mission unique sample capture and storage. Figures 3-2 and 3-3 depict internal features of the SRC, all based off the Stardust experience. 

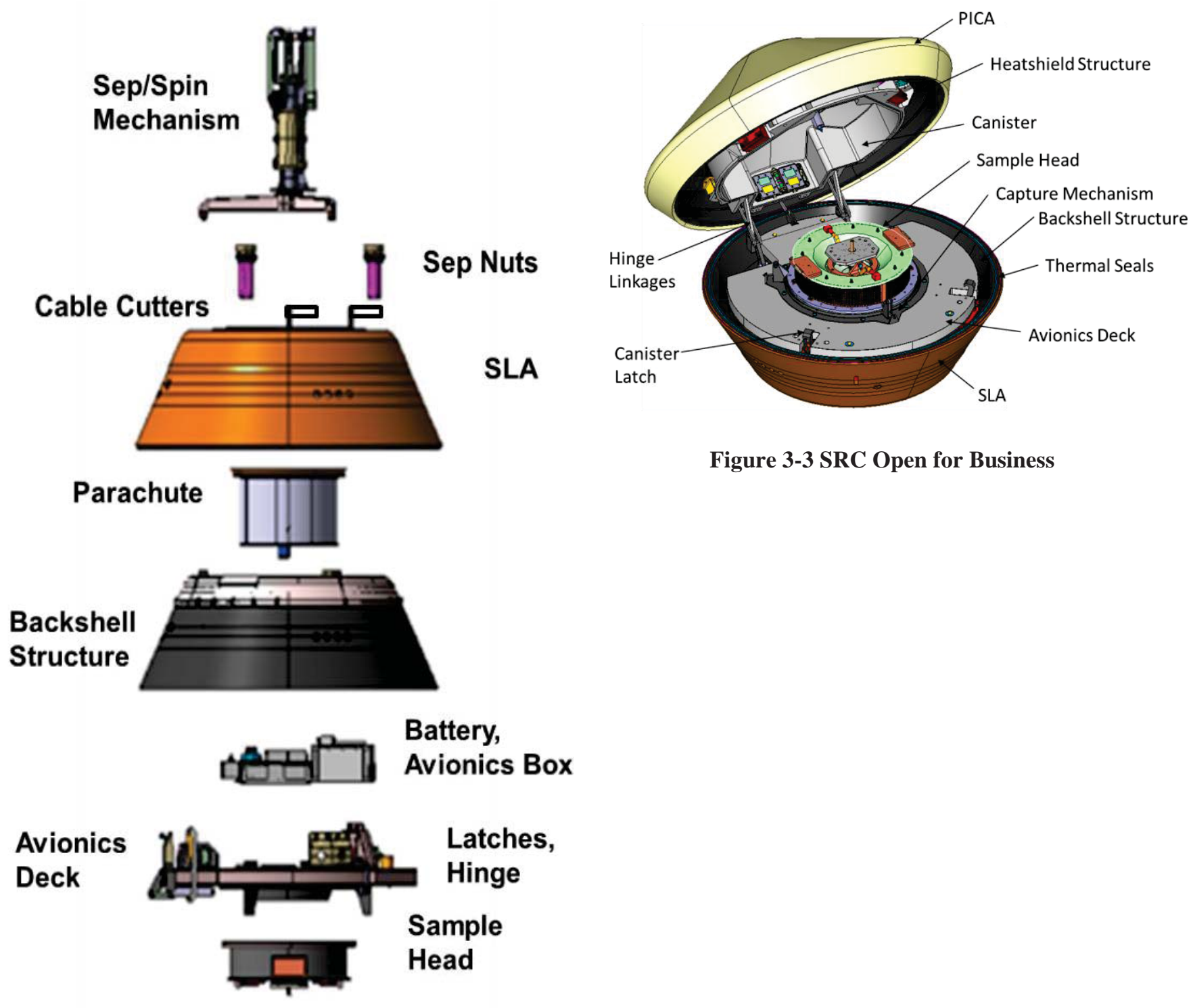

Figure 3-3 SRC Open for Business

Canister
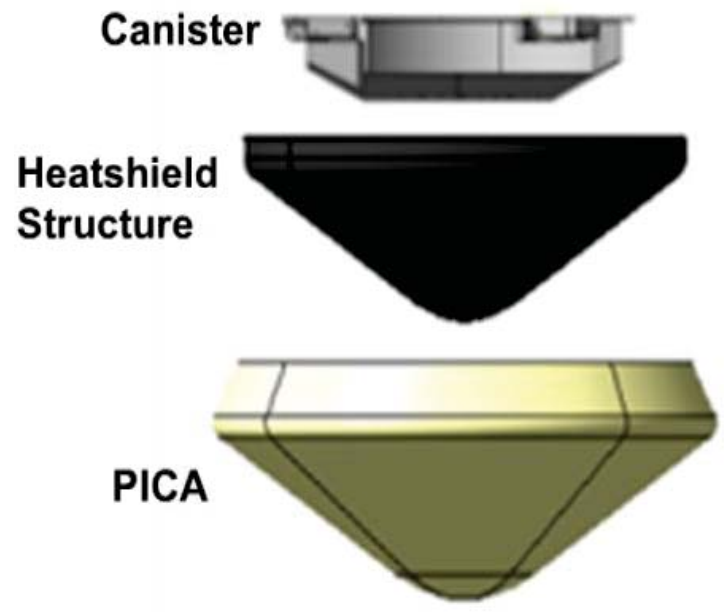

Figure 3-2 SRC Internal Design Features 


\section{SAMPle Collection}

Sample Collection begins in late July 2019 as depicted in Figure 4-6. Before the TAG maneuver, OSIRIS-REx performs a preliminary measurement of spacecraft moment of inertia (MOI) with TAGSAM's Arm deployed. This maneuver provides the baseline for determining the collected sample mass. Following successful rehearsal performance and baseline measurement of spacecraft MOI, the OSIRISREx team commands the spacecraft to perform the sample acquisition. The TAG maneuver follows the transfer orbit, approach, and velocity matching maneuvers as previously rehearsed. Following the Matchpoint maneuver, OSIRISREx approaches the surface along the vector normal to the sampling plane (Figure 4-1) under reaction wheel attitude control, as the instruments continuously collect data to characterize the surface.

At $5 \mathrm{~m}$ from the surface as determined by GN\&C LIDAR, the attitude control system is configured to allow low torque reaction wheel control prior to contact, with large ACS thruster control deadbands to ensure safety during contact. The sampling-event trigger is also armed at this time, and a back-away countdown timer is set based on the time of touch predicted on board using GN\&C LIDAR-derived range data. If surface contact is not sensed before this timer expires, the ACS thrusters fire to back the spacecraft safely away from Bennu. After TAGSAM's firing trigger is armed, surface contact is sensed by a set of two functionally redundant contact triggers that can initiate release of the nitrogen $\left(\mathrm{N}_{2}\right)$ gas for sample collection within the predicted time of touch window. The first contact trigger is acceleration detected by the Inertial Measurement Unit (IMU). The second contact trigger is a compression indicator in the TAGSAM arm. While the Head is in contact with the surface, the Arm absorbs the spacecraft momentum via compression of a spring. This spring holds the Head against Bennu while the $\mathrm{N}_{2}$ sampling gas is discharged, fluidizing the regolith for capture within the Head sample compartment, as shown in Figure 4-2 and depicted in Figure 4-3. The head will capture at least $150 \mathrm{~g}$ of sample (to ensure the $60 \mathrm{~g}$ science requirement is satisfied with the uncertainty in the mass determination) into the bulk sample collector. Simultaneously, the surface pads contact at least $26 \mathrm{~cm}^{2}$ of the surface of Bennu to acquire a surface sample.

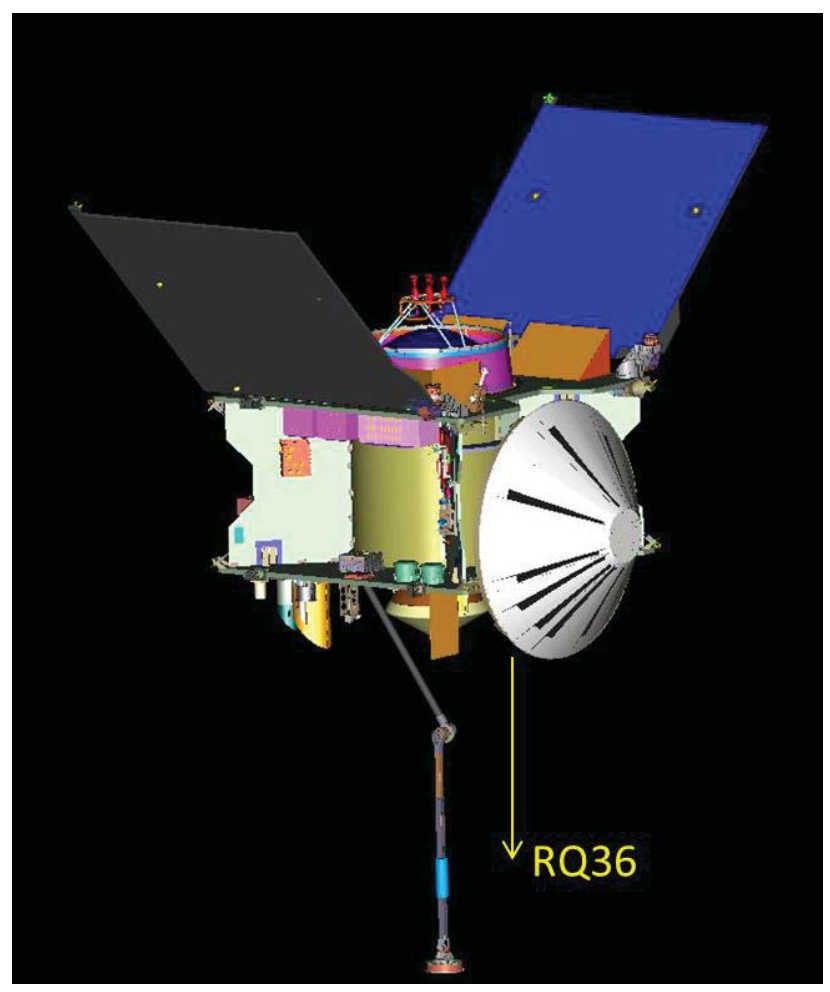

Figure 4-1 Spacecraft Configured for Sample Acquisition

When contact is sensed, the back-away timer is updated with a fixed time to allow sampling to complete before safely backing the spacecraft away from Bennu. When this backaway timer expires, the spacecraft fires ACS thrusters to assist the compliant Arm in reversing the motion of OSIRISREx. All of the ACS thrusters are engaged in a manner to damp rates, remove attitude error induced during the TAG, and safely back the spacecraft away from the asteroid. The back-away maneuver induces $0.7 \mathrm{~m} / \mathrm{sec}$ of $\Delta \mathrm{V}$ away from Bennu. Once the back-away maneuver is complete and the residual rates and attitude error have been removed and the Spacecraft has returned to a Sun-Pointed attitude, control is transferred to the reaction wheels. Once OSIRIS-REx is at a safe distance, the results of the TAG sampling event are evaluated. The Head is then brought into view of the SamCam to visually inspect the surfaces of the Head as shown in Figure 4-4. If this inspection reveals an obstruction that may prevent successfully seating of the Head in the SRC, obstruction removal procedures will be employed. 


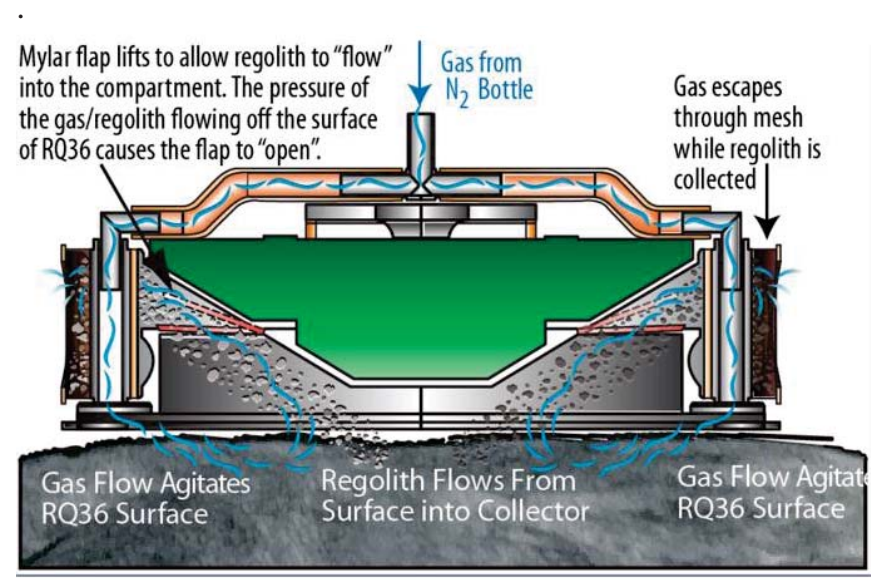

Figure 4-2 Sample Acquisition Mechanism

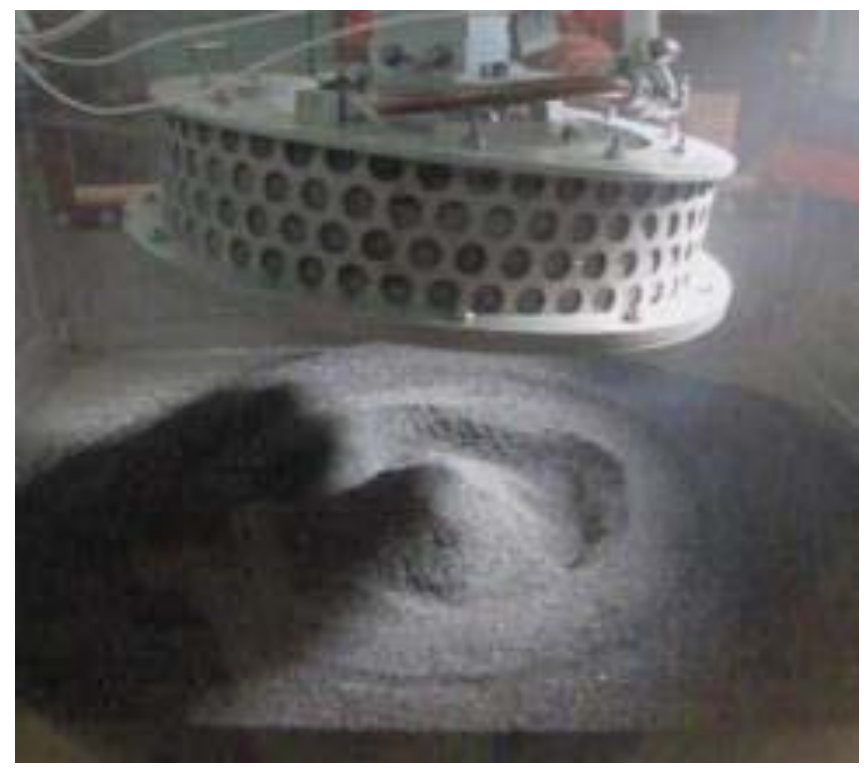

Figure 4-3 Projected footprint on Bennu

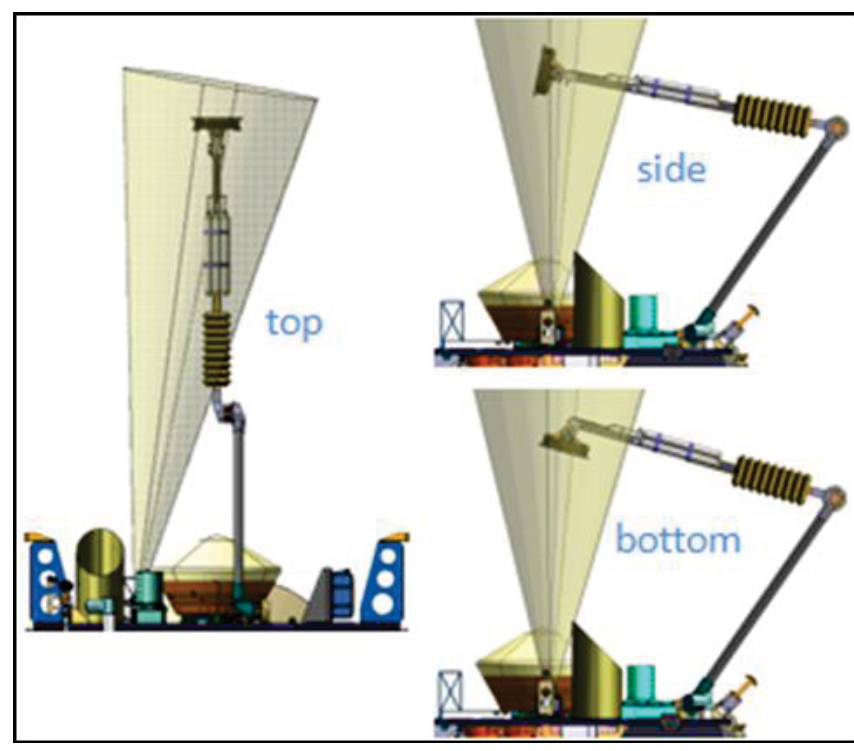

Figure 4-4 Sample imaged for confirmation prior to stowing

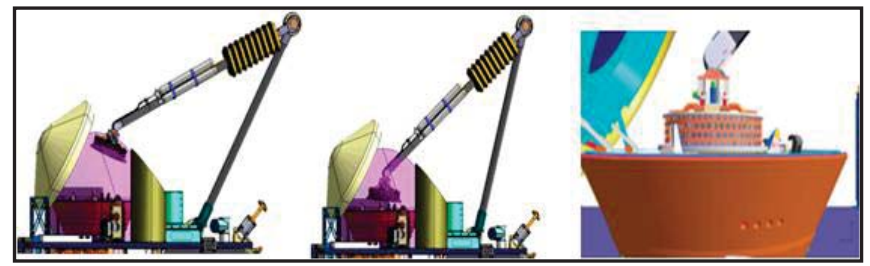

Figure 4-5 The TAGSAM stow sequence

The spacecraft sample mass measurement slews are repeated to determine the change in spacecraft inertial properties from which the mass of the sample is calculated. If the measured mass plus uncertainty ensure $>60 \mathrm{~g}$ was collected, the Principal Investigator (PI), in agreement with NASA HQ, gives consent to stow the sample in the SRC as depicted in Figure 4-5. Otherwise, if an insufficient amount of sample was collected, up to two more sampling maneuvers can be performed. Pending satisfaction of science requirements, the Arm places the sampler into the SRC. Secure attachment is verified with microswitches and StowCam, as well as backdriving the Arm to ensure head is fully locked in place prior to detaching the Head from the Arm. Once the TAGSAM head is detached from the arm, the Arm is stowed back against the side of the Spacecraft, and the SRC is closed and sealed. With the sample safely stowed and ready for return to Earth, OSIRIS-REx enters a period of quiescent ops, with radiation pressure causing the spacecraft to slowly drift away from Bennu until departure for return to Earth in March 2021. Over a year is available between the nominal first sampling attempt in October 2019 and the March 2021 departure, providing ample opportunity to conduct additional sampling attempts, respond to unexpected conditions at Bennu, and deal with spacecraft anomalies. 


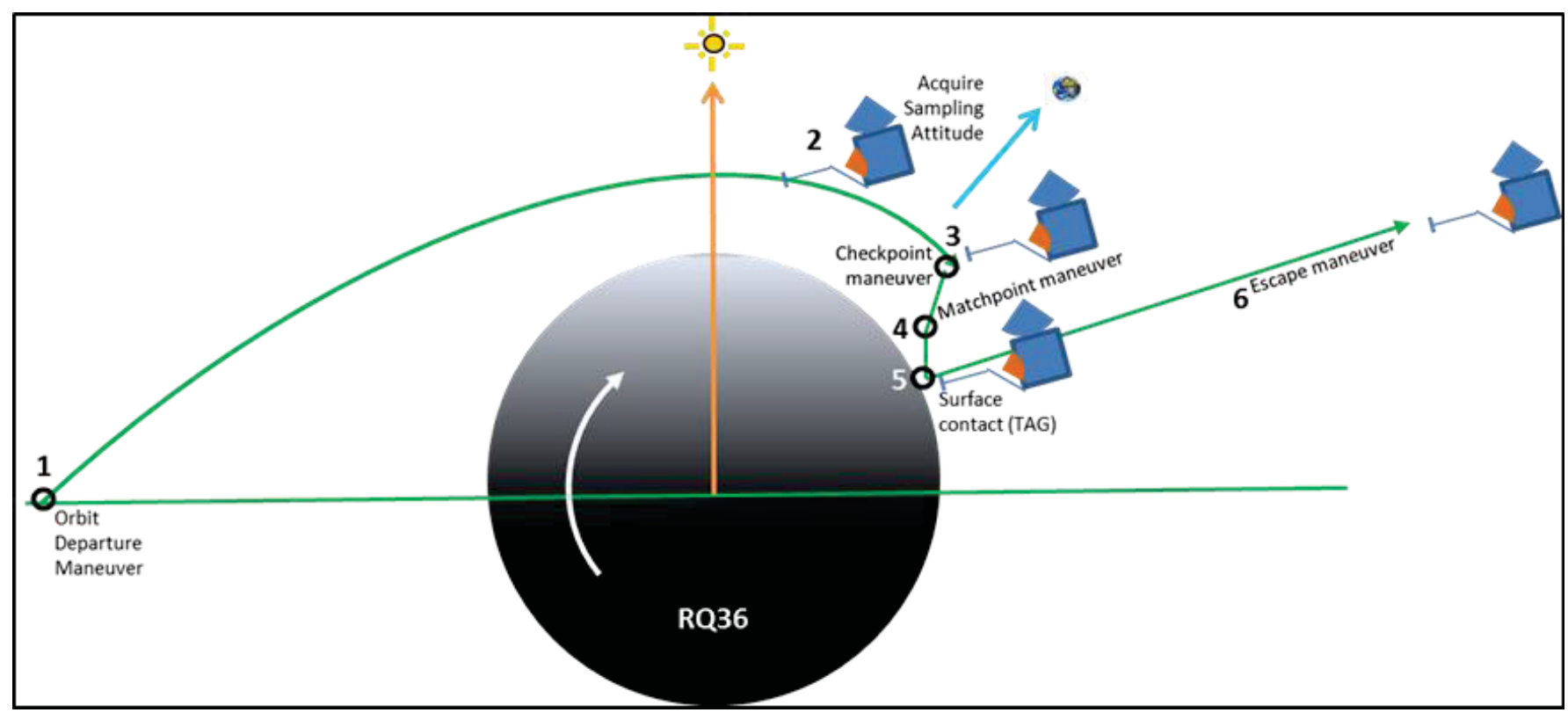

Figure 4-6 Sample Acquisition Sequence of Events 


\section{RETURN CRUISE}

Return Cruise begins with the departure burn in March 2021, 931 days after arrival at Bennu. On March 3, 2021, a 319 m/s $\Delta \mathrm{V}$ departure maneuver sends OSIRIS-REx onto a ballistic return cruise to Earth. No additional large deterministic maneuvers are required to return the SRC to Earth. The trajectory is calculated to safely pass by the Earth if, for any reason, contact with the $\mathrm{S} / \mathrm{C}$ is lost. During the cruise, tracking and TCMs are performed as necessary to precisely target the entry corridor.

Earth and Bennu orbits are depicted below in Figure 5-1.

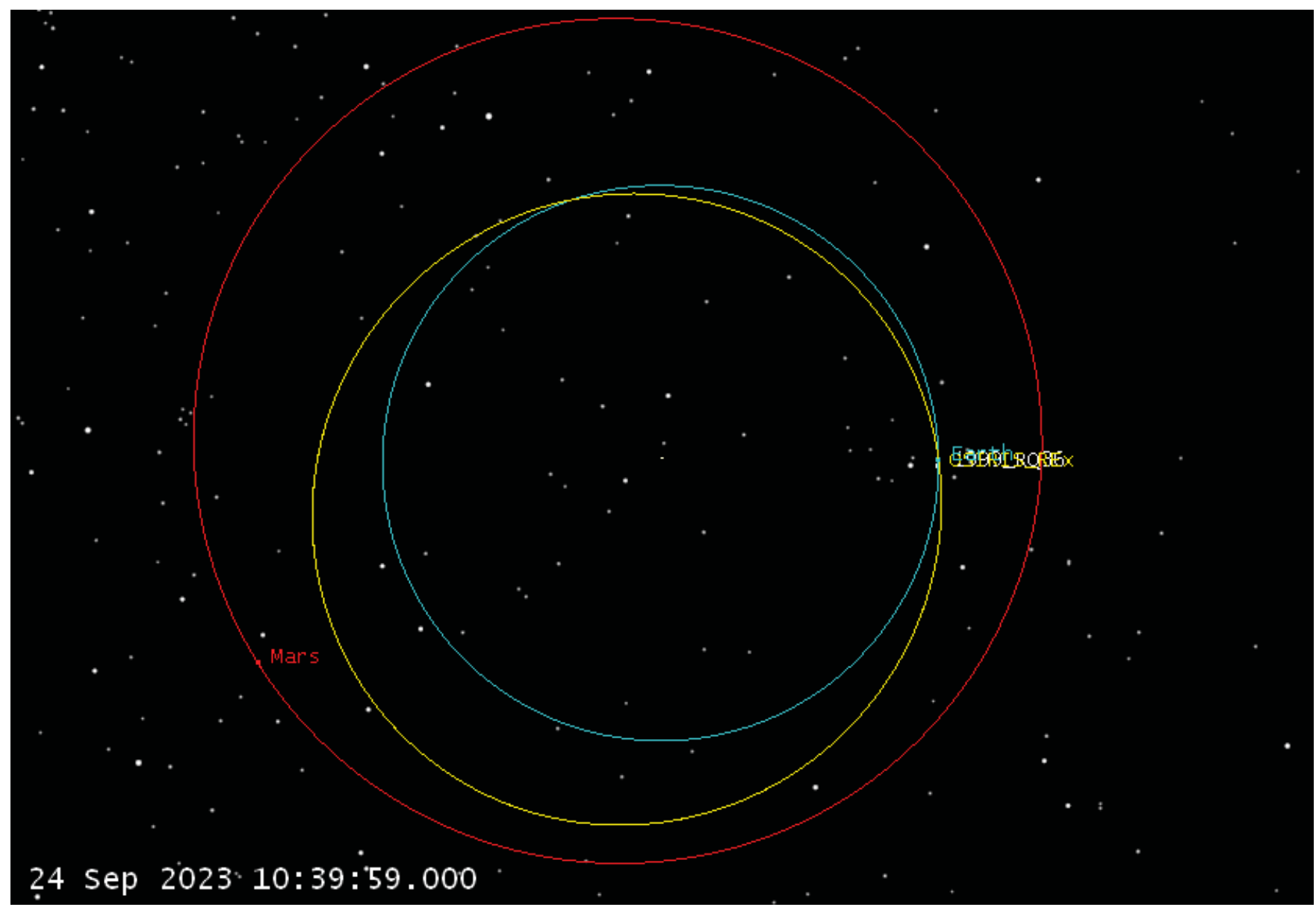

Figure 5-1 Earth (blue), Bennu and OSIRIS-REx (yellow) geometry at Earth return (Mars orbit, red, shown for reference); positions of OSIRIS-REx and Bennu are nearly coincident at this scale

\section{EARTH RETURN AND SRC RECOVERY}

As OSIRIS-REx approaches Earth, the reentry plans are reviewed starting about a year before arrival, and preparations begin. The spacecraft performs approach trajectory correction maneuvers (TCMs) to precisely align with the entry path and to target a minimum altitude of 1,000 $\mathrm{km}$ above Earth as shown in Figure 6-1. The final TCMs target the atmospheric interface $\sim 7$ days prior to entry. After final navigation accuracy checks, including an opportunity for a late correction burn at entry-12 hour, the SRC is released 4 hours prior to atmospheric entry interface.
Twenty minutes later at E-3.7 hour, a divert maneuver raises the $\mathrm{S} / \mathrm{C}$ bus perigee to $250 \mathrm{~km}$ altitude, leaving it in a 1.0 by 0.5 AU solar orbit. Tracking of the spacecraft will continue for $~ 30$ days to aid in long term trajectory analysis. On the final contact, the spacecraft will be placed in safe mode for possible future use, as it has completed its mission. The SRC enters the atmosphere at a nominal entry angle of $-8.2^{\circ}$ at a velocity of $12.2 \mathrm{~km} / \mathrm{s}$ (slightly less than Stardust) on a $62^{\circ}$. azimuth heading that allows communications for the separation and divert events.

The capsule's spin rate provides stability during vacuum flight and early rarified entry. 


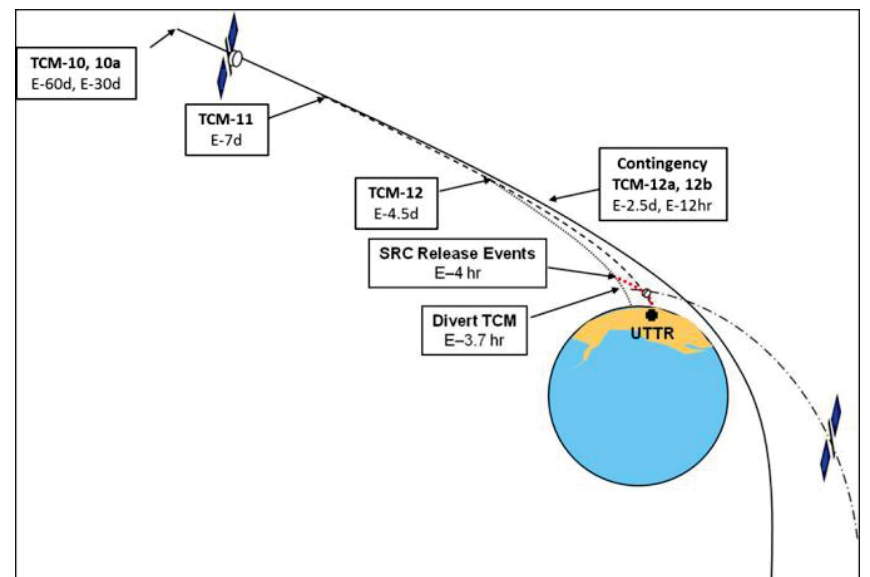

Figure 6-1 Timeline of Earth targeting and SRC release events

As the atmospheric density rises, the vehicle is inherently stable through the max heating regime, where stagnation heating reaches a value slightly less than Stardust. The angle of attack stays well within safe parachute deployment angles through drogue release. The reentry timeline is shown in Figure 6-4. The aeroshell thermal protection system is identical to the Stardust SRC and removes over 99\% of the kinetic energy of the vehicle, protecting the sample from aerodynamic heating and keeping the sample below $75^{\circ} \mathrm{C}$.

A little less than a minute and a half after peak heating, while the SRC is free-falling through the atmosphere, the onboard avionics initiate the parachute sequence as shown in Figure 6.5, with stabilizing drogue deployment at approximately $33 \mathrm{~km}$ altitude. After deploying its main parachute at $3 \mathrm{~km}$ altitude, the SRC lands within a $80 \times 20 \mathrm{~km}$ footprint ellipse (99\%) 15 minutes after entry. On September 24, 2023, the SRC is safely on the ground at the UTTR.

The SRC is tracked with UTTR range radars to within $\sim 10 \mathrm{~m}$ of the landing location with the reentry ground track shown in Figures 6-2 and landing error ellipse shown in Figure 6-3. Once landed, the SRC is recovered and transported to a staging area at UTTR to prepare for transport to JSC.

Air samples are taken at both landing site and staging area to test for SRC outgassing. In addition, relevant soil samples will be taken from the landing site, as well as samples of any other materials the SRC may have come into contact with during landing and recovery. The canister is removed from the SRC and all hardware is transported to the JSC Space Exposed Hardware cleanroom, where the sample canister is opened in the dedicated OSIRIS-REx curatorial facility at JSC. The science team and international cosmochemistry community perform preliminary examination followed by detailed sample analysis. These data provide the final knowledge necessary to achieve the science investigation goals and objectives. Science return from the OSIRIS-REx samples continues for decades as researchers design new instrumentation and develop innovative hypotheses that are tested.

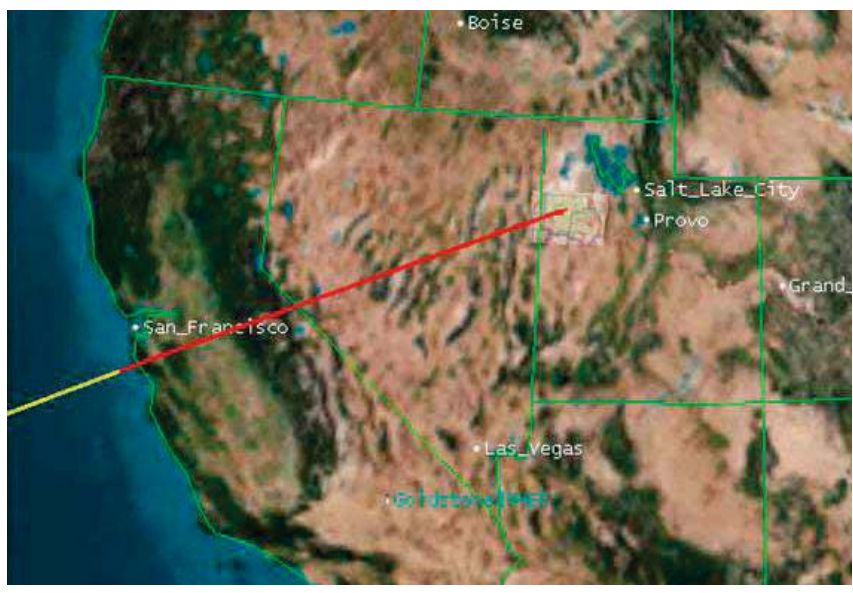

Figure 6-2 SRC Entry Ground Track

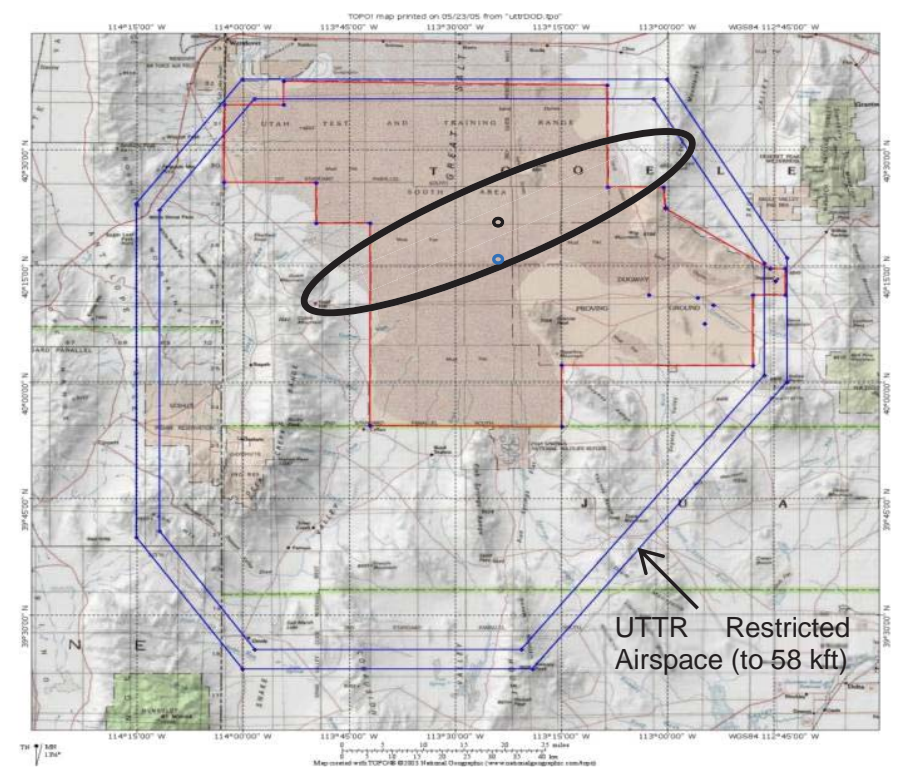

Figure 6-3 Reentry Ellipse at UTTR 


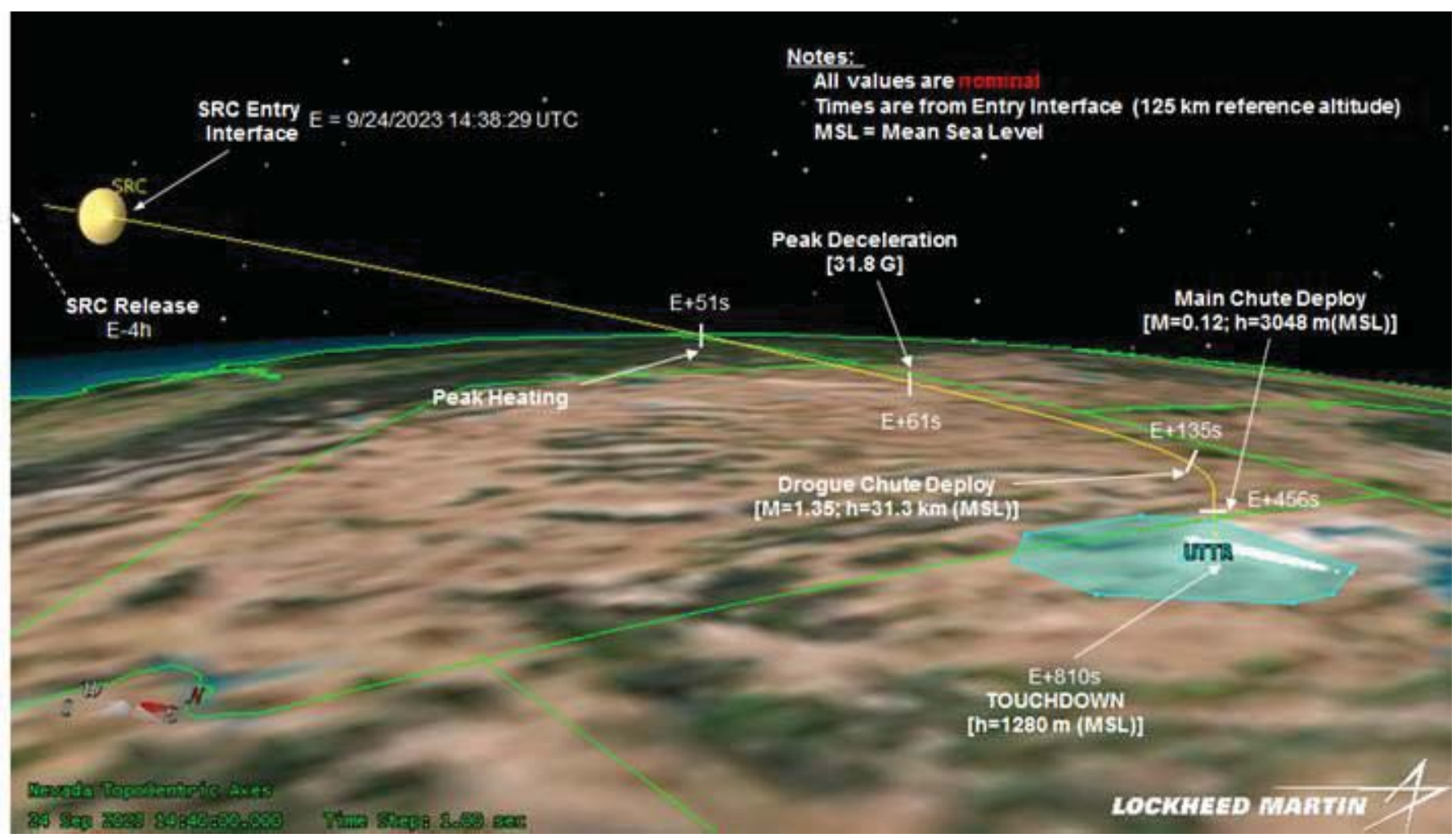

Figure 6-4 Nominal EDL Reentry Timeline

\section{OSIRIS REX PARACHUTE RECOVERY SYSTEM}

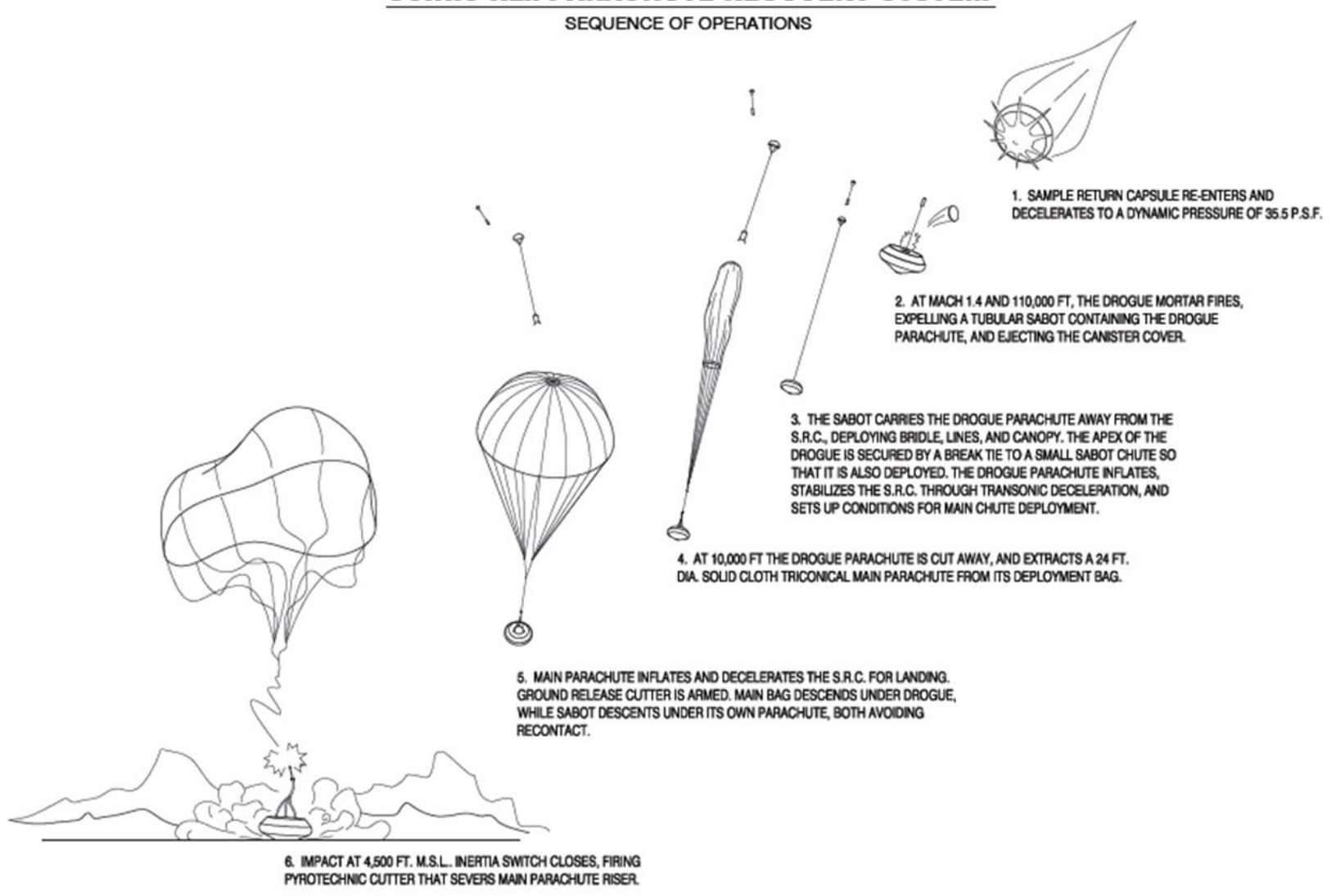

Figure 6-5 Atmospheric Descent and Parachute Deployment Sequence 


\section{SUMMARY}

OSIRIS-REx, the third mission in NASA's New Frontiers Program, will provide the first pristine samples of primitive, carbonaceous asteroid material for analysis in terrestrial laboratories. Analyses of these samples will produce an unprecedented advancement in our scientific knowledge of the initial stages of planet formation and the origin of life.

Launching in September 2016, OSIRIS-REx will return a minimum of $60 \mathrm{~g}$ of pristine bulk regolith and a separate surface sample of fine-grained surface material from nearEarth asteroid (101955) Bennu. The mission will also provide critical data on the Yarkovsky effect which affects the long term orbital evolution of near-Earth asteroids. This knowledge will improve humanity's ability to predict asteroid close encounters with Earth many decades into the future.

OSIRIS-REx brings together all of the elements essential to a definitive asteroid sample return mission - the University of Arizona's leadership in planetary science and experience operating the Mars Phoenix Lander; LM's unique experience in sample-return mission development and operations; Goddard Space Flight Center's (GSFC) expertise in project management, systems engineering, and safety and mission assurance; and KinetX's experience with spacecraft navigation.

The OSIRIS-REx mission is well positioned to meet all mission and science objectives. It has successfully completed all NASA reviews to date including PDR and CDR. While the mission is technically challenging, the high heritage design is considered low risk, and a strong team of commercial and government stakeholders continue to meet or exceed the expectations of this PI led mission.

\section{REFERENCES}

[1] Origins Spectral Interpretation Resource Identification Security-Regolith Explorer (OSIRIS-REx) Project Design Reference Mission and Mission Plan, OSIRIS-REx-OPS-0001, prepared by Ron Mink, NASA/GSFC 